\title{
Algunos problemas con la noción de Sur Global.
}

\author{
Emilio Binaghi
}

Resumen: Este artículo pretende revisar de manera crítica algunas de las tesis centrales a la noción de "Sur Global". Se aborda un resumen de dicha tesis y se propone un abordaje de "alteridades globales" que permite resolver los problemas teóricos de la noción "Sur Global", en particular, la vocación subordinante que se encuentra en el centro de la misma. Así, surge una propuesta vinculada a a noción de traducción y a la figura de lenguaraz como horizonte de vinculación entre los saberes de manera no hegemónica.

Palabras Claves: Sur Global, Alteridades Globales, traducción, lenguaraz

Abstract: This article tries to critically review some of the central theses to the notion of "Global South". A summary of this thesis is approached and an approach of "global alterities" is proposed that allows solving the theoretical problems of the notion "Global South", in particular, the subordinate vocation that is at the center of it. Thus, a proposal arises linked to the notion of translation and to the figure of the lenguaraz as a horizon of connection between knowledge in a non-hegemonic way.

Keywords: Global South, global alterities, translation, lenguaraz

En los últimos años, hemos asistido a la proliferación de una literatura que plantea la existencia de un Sur Global. Cuando hablamos de "Sur Global" hacemos referencia a un lugar geográfico que no podemos encontrar en un mapa, aludiendo más bien a una propiedad relacional del espacio y del poder. El Sur Global no existe por sí mismo sino en relación con un Norte Global, que es el centro del mundo, permitiéndonos integrar a todos los países que se encuentran en una relación de "inferioridad" con ese Norte Global. A partir de una serie de autores que han reflexionado sobre este concepto (entre ellos, quien más ha desarrollado esta idea es Boaventura de Sousa Santos), trataré de desplegar un argumento general sobre el Sur Global y sobre cómo este no lugar geográfico implica una nueva manera de pensar la(s) epistemología(s), la política y el cruce entre ellas. Parte central del argumento gira en torno de los problemas políticos que tiene el propio concepto de "Sur Global". En ese sentido, trataré de esbozar algunos conceptos como los de "alteridades globales" y de "traducción", que son centrales para pensar las condiciones de posibilidad de una relación no jerárquica entre las lenguas y culturas que usualmente se suelen agrupar bajo el paraguas de "Sur Global". 
Boaventura de Sousa Santos plantea la existencia de un Sur Global con el que podemos identificar a todos los países y sociedades que antes habían sido pensados como parte del "Tercer Mundo", o del "subdesarrollo". Cuando se habla de "Sur Global", corremos el eje de donde tradicionalmente se ha lo colocado en la literatura específica, puesto que el propio concepto implica una nueva estrategia política y epistemológica. Ahora no estamos pensando tanto en un desarrollo que aún no ha llegado, pero que ya se encuentra predefinido en el camino que han tomado los países del Norte Global, sino en la posibilidad de un desarrollo autónomo de los países del Sur Global y en sus propios términos. Esta es una distinción clave en el argumento que estoy presentando, puesto que permite cuestionar el pensamiento eurocéntrico que, desde la irrupción del trinomio modernidad-colonialidad-capitalismo, se ha convertido en la norma de lo que podemos considerar el pensamiento racional. Así, el pensar desde un Sur Global permite la emergencia (o mejor dicho, la insurgencia) de una ecología de saberes propios que cuestionan la centralidad del pensamiento racional eurocéntrico, y que a la vez habilitan una tarea central para nosotros, que es repensar la manera en que vinculamos las epistemologías y la política para construir una alternativa real a ese Norte Global opresor. Cabe aclarar que acá tenemos que pensar cómo es posible articular una real ecología de saberes en el Sur Global, cómo podemos generar una vinculación real entre distintos saberes, y a la vez hacer un uso estratégico de ese pensamiento eurocéntrico, para que nos brinde ventajas significativas para la disputa política.

Partir de un Sur Global implica un desafío central, en tanto que se vuelve imperativo establecer una relación no jerarquizada dentro de la ecología de saberes, para poder desarrollar un diálogo intercultural entre diversas constelaciones epistemológicas. Aquí, la apuesta de Enrique Dussel nos brinda un indicio de cómo se podría articular ese diálogo. En "Una nueva edad mundial en la historia de la Filosofía" este autor plantea la posibilidad del diálogo intercultural a través de un diálogo interfilosófico, a partir de una estructura neurológica compartida por toda la humanidad. Así, esa estructura compartida, en conjunto con los núcleos problemáticos universales, establecen una supuesta base objetiva para el diálogo intercultural e interfilosófico. Señalar los límites de esta mirada con la propuesta que hacemos parece buscar matices en donde no hace falta, pero se 
vuelve necesario plantear que esta base neurológica que propone Dussel termina siendo prisionera del propio modelo eurocéntrico que dice combatir. Sin duda, la mirada cientificista desplegada en este punto por Dussel no es respetuosa con la propuesta de una ecología de saberes, ya que, implícitamente, supone que la medicina occidental que se encuentra en la base de la neurociencia, es de un orden superior a otros saberes que podrían dar una explicación similar. Es rescatable un punto central en la argumentación dusseliana que no debe ser dejado de lado. La posibilidad del diálogo intercultural e interfilosófico que propone tiene un logro no menor, que es discutir este diálogo, en términos de traducibilidad. Con esto quiero decir que Dussel señala que el diálogo sólo es posible en tanto que se traduzca (atendiendo aquí al uso particular que Dussel hace del término y que se encuentra directamente relacionado con su propia concepción de la hermenéutica) de una lengua (y una cultura) del Sur a otra del Sur. Esta estrategia de traducción logra resolver un problema clave en las relaciones interfilosóficas: la de depender de las lenguas (y culturas filosóficas) de los países del Norte Global para que funcionen como punto de contacto y espacio de intertraductibilidad e intercambio entre las diversas traducciones del Sur, el cual sigue quedando en un lugar subordinado a las lenguas y filosofías del Sur, que no pueden intercambiar entre ellas sin un paso previo por las filosofías del Norte.

Por fuera de este desvío dusseliano, querría pensar una intuición que guía este trabajo. La concepción del Sur Global, que resulta una concepción sin duda valiosa en tanto que herramienta de unidad entre los países periféricos, conlleva una serie de problemas que no puedo dejar de señalar. En primer lugar, en consonancia con lo que plantea Wainaina en "How to write about Africa", hablar de "Sur Global" implica homogeneizar al sujeto que trata de nombrar, suprimiendo diferencias centrales entre los países que forman parte de este "Sur global", y que pierden su especificidad en pos de ser nombrados. La idea de que el carácter periférico de dichas sociedades las coloca en el mismo plano no permite pensar las especificidades que llevaron a dicha condición periférica. Así, definir por la negativa, aún desde una posición que podemos considerar como progresista, termina siendo funcional a un discurso simplificador de las alteridades sociales y políticas que habitan los mundos no centrales del planeta, y supone caer en el mismo error que señala Edward Said cuando se refiere al modo en que fue acuñado el 
término "orientalismo" para dar cuenta de las características propias de las sociedades que el Occidente europeo consideró como otredades respecto de sí.

Esta operación homogeneizadora, que bienintencionadamente ponen en juego una serie de autores, termina generando el mismo problema epistemológico que busca saldar. Cualquier teoría que busque abarcar la totalidad de ese Sur Global, cae en el mismo problema que otros intentos teóricos por encontrar una teoría capaz de dar cuenta de toda la realidad. Parece que el reduccionismo y el aplanamiento de las diferencias son los precios a pagar por una teoría ( $\mathrm{y}$ una práctica) que buscan una explicación omnicomprensiva. Así, desde una mirada reivindicativa de los países periféricos, se termina adoptando una lógica propia del pensamiento eurocéntrico.

Estos desafíos, apenas esbozados, me llevan a plantear una doble estrategia. Por un lado, a partir del concepto de Rita Segato sobre "alteridades históricas", propongo pensar la noción de "alteridades globales" para nominar estas opciones que no han podido surgir plenamente por la opresión, directa o indirecta, de los países centrales. Creo que este concepto da cuenta de las diferencias específicas de cada una de las sociedades, sean del tipo que sean; permite dar cuenta de la relación siempre compleja que dichas alteridades globales establecen unas con otras. Relaciones no necesariamente armoniosas y no exentas de conflictividad, puesto que el carácter de alteridad no quita la posibilidad de un ejercicio colonial (colonial en tanto que subyugador de culturas y sociedades-otras, con las cuales se comporta como una potencia colonial). Asimismo, al pensar en términos de "alteridades globales", se lograría conformar un concepto capaz de dar cuenta de unidades sociales menos atadas a categorías economicistas, marca de origen del concepto de "Sur Global", y pasibles de quedar subsumidas en unidades de nominación nacionales más amplias. Este es un riesgo demasiado habitual, más aun si el foco es puesto en el interior de los estados, que han buscado sistemáticamente operar como fuerzas homogeneizadoras hacia dentro de las diversas alteridades que los habitan.

En segundo lugar, la idea de "alteridades globales" permitiría alcanzar un marco más igualitario para abordar propuestas como la de Boaventura de Sousa Santos, en tanto que una ecología de saberes (y la sociología de las ausencias que implica) permite pensar mejor la relación entre los saberes desplegados por las distintas alteridades globales y cómo las mismas pueden brindar herramientas para el diseño de estrategias políticas de 
resistencia, de alcance global. Así, las "alteridades globales" como concepto hace posible pensar en una relación más igualitaria entre los distintos saberes, escapando al problema de la subordinación de un conjunto de saberes a otro.

Con esto quiero plantear que es necesario establecer una relación no jerárquica entre los saberes producidos por las alteridades globales, a fin de lograr "préstamos" conceptuales entre las mismas, con el objetivo político de surgir, de insurgir, frente al Norte Global. Esta propuesta de préstamos conceptuales sólo es posible en tanto que logremos dar con un concepto de "traducción" que escape a los problemas que Dussel señala: el problema del pensamiento eurocéntrico como lengua franca de la ecología de saberes, para construir una traducción, un diálogo Sur-Sur.

Una breve nota final para señalar algunas características que, según creo, deberíamos buscar en el diálogo/traducción Sur-Sur. Para dar cuenta de las especificidades locales que tienen dichos conceptos, y lograr apoyarnos en ellos para intervenir políticamente en los propios contextos, es necesario concebir la traducción, en una modulación gramsciana, más como producción que como mera transliteración de palabras.

En este sentido, pienso que la figura -ambigua y compleja- del lenguaraz, tal como la trabaja Guillermo David en El indio deseado. Del dios de la Pampa al indiecito gay es un ejemplo del tipo de concepto que estoy planteando aquí. El lenguaraz habita dos mundos; traduce, reescribiendo en otra lengua lo dicho y planteado en dos mundos distintos, ${ }^{1}$ pero no como una traducción ociosa que busca simplemente intercambiar literaturas e ideas por el mero placer de hacerlo sino, en sintonía con la apropiación

1 La figura del lenguaraz, que es visible en los relatos sobre el mundo indio poscolonial, siempre emerge al momento de la negociación con el hombre blanco, con el poder estatal de una alteridad global que se quiere imponer sobre una cultura considerada inferior. Una de las múltiples ambigüedades y paradojas que habitan al lenguaraz es que, en muchas ocasiones, eran hombres blancos que habían decidido cruzar la frontera y vivir en el mundo del indio, en un gesto cruziano fundante para el rol de la traducción (y de los traductores) que estoy pensando. En algún punto, reivindico la positividad que Todorov le endilga a Malinche en La conquista de América, en tanto que puente cultural entre España y América. Dicho esto, no hay que soslayar, como el propio Todorov indica, dos cuestiones. En primer lugar, no se debe minimizar el rol como facilitadora, en el caso de Malinche, de la conquista, como aliada clave de Córtes y sin la cual, a conquista de México no hubiera acontecido. Por otra parte, Malinche, y su historia posterior, son parte de un alegato por el mestizaje cultural que va en sentido contrario de la idea aquí discutida. Las tesis del mestizaje, tan caras a Gilberto Freyre para poner un ejemplo, no dejan de ser una máscara alegre, colorida, que oculta una historia de dominación y subordinación racial y política. 
caníbal que plantea Julio Cabrera, como "pensacción"; es decir, como combinación del pensamiento y la acción para la liberación epistemológica del(de los) Sur(es). Así, la traducción se convierte en la vía para la reconversión productiva de saberes y experiencias de las alteridades globales, a fin de diseñar políticas activas de resistencia contra un Norte Global que se solaza en no dejarnos surgir como pueblos libres.

\section{Referencias:}

CABRERA, Julio. "Esbozo de una introducción al pensamiento desde "América Latina". (más allá de las “introducciones a la filosofía”) en Willames Frank y Alberto Vivar Flores Problemas do pensamento filosófico na América Latina, Phillos,Goiânia, 2018.

DAVID, Guillermo; El indio deseado. Del dios de la Pampa al indiecito gay, Las Cuarenta, Buenos Aires, 2011.

DUSSEL, Enrique; "Una nueva edad mundial en la historia de la Filosofía", en Dussel, Enrique; Filosofías del Sur. Descolonización y transmodernidad. Akal, Buenos Aires, 2016.

WAINAINA, Binyavanga; How to Write about Africa citado de https://www.bu.edu/africa/files/2013/10/How-to-Write-about-Africa.pdf

Recebido e aprovado em março de 2020 * Esse trabalho é licenciado pela Creative Commons Attribution-NonCommercial 4.0 International License 